\title{
Ultrasound- and hemicellulase-assisted extraction increase $\beta$-glucosidase activity, the content of isoflavone aglycones and antioxidant potential of soymilk
}

\author{
Mariah Benine Ramos Silva ${ }^{a}$, Heloisa Gabriel Falcão ${ }^{a}$, Louise Emy Kurozawa ${ }^{\mathrm{b}}$, \\ Sandra Helena Prudencio ${ }^{\mathrm{a}}$, Adriano Costa de Camargo ${ }^{\mathrm{a}, \mathrm{c}^{*} \text {, }}$ \\ Fereidoon Shahidid and Elza Iouko Ida ${ }^{\mathrm{a}^{*}}$
}

\begin{abstract}
aLondrina State University, Department of Food Science and Technology, 86051-990 Londrina, Parana State, Brazil
${ }^{b}$ Department of Food Engineering, Faculty of Food Engineering, University of Campinas, Campinas, SP, Brazil

'Departamento de Ciencias Vegetales, Facultad de Agronomía e Ingeniería Forestal, Pontificia Universidad Católica de Chile, Casilla $306-$

22, Santiago, Chile

dDepartment of Biochemistry, Memorial University of Newfoundland, St. John's, NL A1B 3X9, Canada

* Corresponding author: Adriano Costa de Camargo and Elza Iouko Ida, Londrina State University, Department of Food Science and Technology, 86051-990 Londrina, Parana State, Brazil., E-mail: adrianoesalq@gmail.com, elida@uel.br
\end{abstract}

DOI: $10.31665 /$ JFB.2019.6191

Received: June 25, 2019; Revised received \& accepted: June 28, 2019

Citation: Silva, M.B.R., Falcão, H.G, Kurozawa, L.E, Prudencio, S.H, de Camargo, A.C, Shahidi, F., and Ida, E.I. (2019). Ultransound- and hemicellulase-assisted extraction increase $\beta$-glucosidase activity, the content of isoflavone aglycones and antioxidant potential of soymilk J. Food Bioact. 6: 140-147.

\begin{abstract}
Soymilk is consumed as such or used in the preparation of tofu, fermented soymilk, and soy yogurt, among others. In this study, hemicellulase-assisted extraction (HAE) and ultrasound-assisted extraction (UAE) were used during soymilk manufacturing. The activity of endogenous $\beta$-glicosidases was increased 1.3 and 1.5 -fold in soymilk produced upon HAE and UAE, respectively. Likewise, the concentration of isoflavones in the aglycone form increased 1.7 and 2.4 times in samples subjected to HAE and UAE, respectively. UAE, but not HAE, rendered an equimolar conversion from conjugated isoflavones to their respective aglycones. In addition, HAE and UAE showed higher antiradical activity towards ABTS radical cation and peroxyl radical, compared to those of the control. The aglycone isoflavones/conjugated isoflavones (aISO/cISO) ratio correlated with the antiradical activity, thus suggesting that the soybean industry may use this new quality index to indicate the antioxidant potential of products. Food formulations containing soymilk with high aISO/cISO ratio may translate to higher oxidative stability of the final product. Likewise, the higher absorption of aglycone isoflavones compared to that of their conjugated counterparts is well recognized. Therefore, high alSO/cISO ratio of soymilk and/or its products may provide a better antioxidant protection under physiological conditions.
\end{abstract}

Keywords: Soybean; Hemicellulase from Aspergillus niger; Sonication; Phenolic antioxidants; Antioxidant potential.

1. Introduction

Soymilk has been recognized by its high protein content. It can be consumed as such or used in the preparation of tofu, fermented soymilk, soy yogurts, among others. Production and commer- cialization of soymilk has steadily increased in recent years as an option for vegetarians and vegans, but also due to numerous evidences supporting its health benefits, attributed mainly to its phytochemicals, especially phenolic compounds such as flavonoids (Toro-Funes et al., 2014). The transformation of flavanones 
to isoflavone is a two step process which involves the action of 2-hydroxyisoflavanone synthase and 2-hydroxyisoflavanone dehydratase (Veitch, 2007), although the action of isoflavone synthase has also been noted in describing the overall reaction (de Camargo et al., 2018; Veitch, 2007).

As plant food secondary metabolites, flavonoids and other phenolic compouds are related to the plant defense against pests and pathogens as well as biotic and abiotic stress. As for the possible health benefits to humans, isoflavones have been widely investigated due to their association with reduced risk of several chronic ailments such as cardiovascular and Alzheimer diseases, some types of cancer, and type 2 diabetes, among others. Furthermore, soybean isoflavones may also alleviate menopausal symptoms (de Camargo et al., 2019b; Kalaiselvan et al., 2010).

The literature has described the existence of four groups of isoflavones, namely, aglycones (genistein, daidzein, and glycitein), $\beta$-glucosides (genistin, daidzin, and glycitin), acetylglucosides (acetylgenistin, acetyldaidzin, and acetylglycitin), and malonylglucosides (malonylgenistin, malonyldaidzin, and malonylglycitin). Therefore, depending on the cultivar and processing method employed, there may be twelve different isoflavones in soybean and its processed products (Falcão et al., 2018).

Digestion and absorption of isoflavones are influenced by their chemical structures (Shahidi and Peng, 2018) and, it has been accepted that, conjugated isoflavones are poorly absorbed in the small intestine. In fact, according to a human study by Izumi et al. (2000), conjugated isoflavones are absorbed more slowly and in lower amounts than their corresponding aglycones, which has been explained by their hydrophilic nature and higher molecular weight. Hence, to increase their bioaccessibility and render greater bioactivities, conjugated isoflavones should be hydroysed into their corresponding aglycones. There is a wealth of evidence supporting the improvement of soy protein bioactivity upon enzymatic hydrolysis (Farzamirad and Aluko, 2008). Likewise, the procurement of soybean products with higher contents of aglycone isoflavones could be helpful to the user industries.

Endogenous $\beta$-glucosidases present in soybeans catalyze the hydrolysis of glycosidic bonds from conjugated isoflavones thus generating their respective aglycones (Yoshiara et al., 2018a). Furthermore, thermal treatment, fermentation, and ultra-high pressure homogenization are examples of other methods that have been tested to improve the recovery of isoflavone aglycones (Andrade et al., 2016; Baú et al., 2015; Fernandes et al., 2017; Handa et al., 2014; de Lima and Ida, 2014; Toro-Funes et al., 2014).

Enzyme-assisted extraction to improve the recovery of phenolic compounds has been used as an alternative to alkali treatment. Thus, cell wall degrading enzymes have shown greater potential than those of non-specific proteases (de Camargo et al., 2016). The cell wall of soybean is mainly composed of hemicellulose, followed by pectin and cellulose (Riaz, 2006). Hemicellulases (e.g. $\alpha$-glucuronidase, $\alpha$-arabinofuranosidase, arabinase, endo-mannanase, $\beta$-mannosidase, acetyl xylan esterase, and feruloyl xylan esterase), which depolymerize hemicelluloses, are usually of microbial origin (Juturu and Wu, 2012). However, the cost associated with enzyme purchase and treatment may sometimes be prohibitive. Therefore, technologies such as ultrasound-assisted extraction could be advantageous with respect to enzymatic treatment by enhancing the observed effects. Propagation waves, which results in compression/expansion cycles, are formed in the solvent system upon ultrasound application. During the ultrasound propagation in the solvent system, a decrease in the pressure with consequent generation of bubbles, also known as cavitation, takes place. These microbubbles expand until they collapse next to the surface of the cell wall of the plant material. High pressure and temperature regions are among the factors interacting with the physical integrity of the cell walls, which may facilitate the release of bioactive compounds linked to them (Arvayo-Enríquez et al., 2013; Nadar and Rathod, 2017). Rao and Rathod (2015) reported that ultrasound increases the permeability of the cell wall to the solvent. Therefore, in food systems, an improved interaction of water with the biomolecules present in the test material may be expected.

Enzyme and ultrasound treatment may increase the activity of endogenous $\beta$-glucosidase, enhance the conversion of conjugated isoflavones into their aglycone forms as well as affecting the antioxidant potential of soymilk. A recent study by Preece et al. (2017) demonstrated that ultrasound treatment improves protein extraction yield during soymilk production. However, to the best of our knowledge, the effect of ultrasound on the identities and concentration of isoflavones as well as the outcome in terms of antioxidant properties have not been addressed. Furthermore, we consider this to be the first report investigating the isoflavone profile, activity of endogenous $\beta$-glucosidase and antiradical activity of soymilk as affected by hemicellulase-assisted extraction. Therefore, the effects of both treatments were evaluated for the aforementioned parameters. The results presented here are important for the soymilk industry and may influence the application of soymilk in food formulations. Hence, this contribution will provide means to anticipate potential benefits to the consumers due to the strategies employed.

\section{Materials and methods}

\subsection{Samples, chemicals and solvents}

Lipoxygenase-free BRS 257 soybean samples [Glycine Max. (L.) Merr], crop year 2016, were kindly donated by SL Alimentos (Londrina, Paraná, Brazil). Acetylglycoside (acetyldaidzin, acetylgenistin, and acetylglycitin) and malonylglycoside isoflavones (e.g., malonyldaidzin, malonylgenistin, and malonylglycitin) were procured from Wako Pure (Osaka, Japan). $\beta$-Glycoside isoflavones (daidzin, genistin and glycitin) and all aglycone standards (daidzein, genistein and glycitein) were purchased from Sigma-Aldrich (St. Louis, MO, USA). Folin-Ciocalteu, gallic acid, $p$-nitrophenyl $\beta$-D-glucopyranoside ( $p$-NP), 6-hydroxy-2,5,7,8tetramethylchromane-2-carboxylic acid (trolox), 2,2'-azino-bis(3ethylbenzothiazoline-6-sulphonic acid) diammonium salt (ABTS), 2,2'-azobis(2-methylpropionamidine) dihydrochloride (AAPH), and hemicellulase from Aspergillus niger (0.3-3.0 unit mg-1 solid) were purchased from Sigma-Aldrich (St. Louis, MO, USA). The remaining chemicals and solvents were of analytical or chromatographic grade and were used as received.

\subsection{Soymilk preparation}

Soybean samples were mixed with water at 1:3 (w/v) and macerated at $5{ }^{\circ} \mathrm{C}$ for $14 \mathrm{~h}$. The water was drained and the grains were homogenised for 2 min using a M07 Power Mixer (Mondial, Barueri, SP, Brazil) (Baú and Ida, 2015). The homogenate was used to evaluate the effect of hemicelulase- and ultrasound-assisted extraction. To the homogenate, $1.5 \%$ hemicelulase was added while stirring for $3 \mathrm{~min}$ at $37^{\circ} \mathrm{C}$ using a Dubnoff TE-053 water bath (Tecnal, Piracicaba, Brasil). Ultrasound-assisted extraction was evaluated with a Q700 QSonica equipment (Newtown, CT, USA) operated at $20 \mathrm{kHz}$ and $50 \mathrm{~W} \mathrm{~cm}^{-2}$ ). The treatment took 3 min and a $1 / 2^{\prime \prime}(13 \mathrm{~mm})$ diameter titanium probe was used. A sample devoid of enzyme or ultrasound treatment was kept as a control. The 
homogenates were filtered using a 150 mesh cloth. The soymilk so obtained was frozen in a MDF-U32V Sanyo ultra-low temperature freezer (Wood Dale, IL, USA), lyophilized using an Alpha 1-2 LD Plus freeze dryer (Martin Christ, Germany) and stored at $-18{ }^{\circ} \mathrm{C}$ until further analysis.

\subsection{B-Glucosidase activity}

Endogenous $\beta$-glucosidases were extracted according to de Lima and Ida (2014). The extracts were centrifuged (794 $g$ ) for 15 min at $4{ }^{\circ} \mathrm{C}$ using an Eppendorf 5804R equipment (Eppendorf, Hamburg, Germany). The upper layer was collected and used for $\beta$-glucosidase activity determination (Matsuura and Obata, 1993) as described by de Lima and Ida (2014). The activity unit (AU) is defined as the quantity of $\beta$-glucosidase required to release $1 \mu \mathrm{mol}$ of $p$-nitrophenyl- $\beta$-D-glucopyranoside per min. The $\beta$-glucosidase activity was expressed as AU. $\mathrm{g}^{-1}$ on a dry weight basis.

\subsection{Isoflavone profile and antioxidant potential}

\subsubsection{Extraction procedure}

Ground soybean, control soymilk as well as the soymilk obtained under hemicelulase- and ultrasound-assisted extraction were lyophilized and defatted with hexane $(1: 20, \mathrm{w} / \mathrm{v})$ for $1 \mathrm{~h}$ using a MA 140/CFT shaker (Marconi, São Paulo, Brazil). Defatted samples $[1: 20(\mathrm{w} / \mathrm{v})]$ were extracted with ultrapure water-ethanol-acetone $(1: 1: 1, \mathrm{v} / \mathrm{v} / \mathrm{v})$ according to Yoshiara et al. (2018a). This mixture was agitated every 15 min using a vortex for $1 \mathrm{~h}$ after which it was ultrasonicated for 15 min using a USC1450 Unique sonicator (Unique, Indaiatuba, Brasil). The supernatants were collected after centrifugation at $794 \mathrm{~g}$ for $15 \mathrm{~min}$ at $4{ }^{\circ} \mathrm{C}$ using an Eppendorf 5804 R centrifuge (Hamburg, Germany). The isoflavone-rich extracts so obtained were used for isoflavone profiling and antioxidant activity determinations.

\subsubsection{Identification and quantification of isoflavones by ultra performance liquid chromatography (UPLC)}

The UPLC method described by Falcão et al. (2018) was used for identification and quantification of isoflavones. As each isoflavone has a different molecular weight, the results are expressed as $\mu \mathrm{mol}$ of isoflavone per gram of sample $\left(\mu \mathrm{mol} \cdot \mathrm{g}^{-1}\right)$ on a dry weight basis. This would allow to make an accurate discussion on the molecular conversion of conjuated isoflavones to their corresponding aglycones.

\subsubsection{Scavenging activity towards ABTS radical cation}

The scavenging activity towards ABTS radical cation (Re et al., 1999) was carried as described by Handa et al. (2016). The ABTS radical cation was generated by reacting ABTS $(7 \mathrm{mM})$ with potassium persulphate $(2.45 \mathrm{mM})$. Both chemicals were prepared in distilled water and kept in the dark for $16 \mathrm{~h}$ at $25^{\circ} \mathrm{C}$. This stock solution was diluted with phosphate buffered saline ( $20 \mathrm{mM}, \mathrm{pH} 7.4)$ to an absorbance of $0.70 \pm 0.02$, which was read at $730 \mathrm{~nm}$ using a Biochrom Libra S22 spectrophotometer (Biochrom, Cambridge, England). The reaction was carried out with $10 \mu \mathrm{L}$ of sample and $4 \mathrm{~mL}$ of ABTS radical cation for 6 min after which the absorbance was read at $730 \mathrm{~nm}$ using the same equipment. The ABTS radical

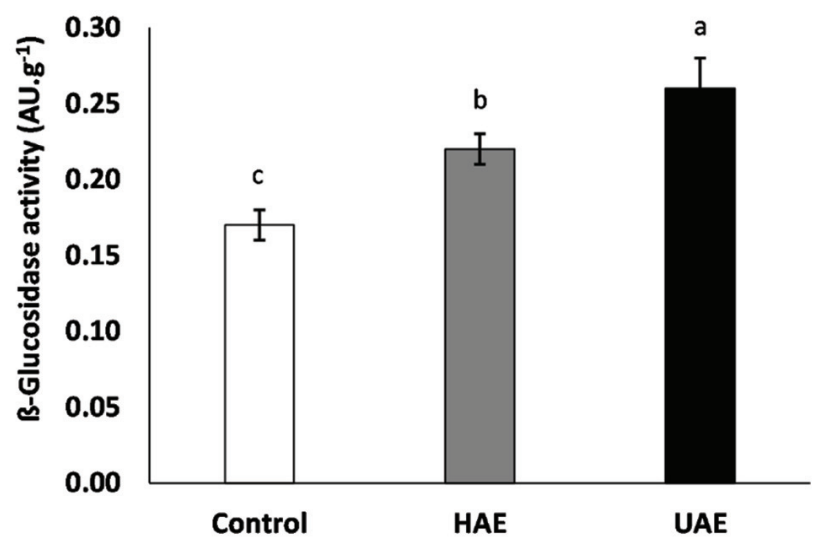

Figure 1. Effect of hemicellulaseassisted extraction (HAE) and ultrasoundassisted extraction (UAE) on the activity of endogenous ß-glucosidase. Means with different letters show difference among treatments $(p<0.05)$.

scavenging activity was expressed as trolox equivalents per gram of sample on a dry weight basis.

\subsubsection{Scavenging activity towards peroxyl radical}

The scavenging capacity towards peroxyl radical was determined following the procedure described by Melo et al. (2015). Peroxyl radical oxidizes fluorescein, therefore changes in the fluorescence decay were recorded. The reaction was carried out in a microplate by mixing the samples $(30 \mu \mathrm{L}), 508.25 \mathrm{nM}$ fluorescein $(60 \mu \mathrm{L})$ and $76 \mathrm{mM}$ AAPH $(110 \mu \mathrm{L})$. All solutions prepared in $75 \mathrm{mM}$ potassium phosphate buffer ( $\mathrm{pH} 7.4$ ), which was also used as a blank. The reaction was monitored every $\min$ for $2 \mathrm{~h}$ at $37^{\circ} \mathrm{C}$. The microplate reader (Molecular Devices, LLC, Sunnyvale, CA, USA) was set at 485 and $528 \mathrm{~nm}$ for excitation and emission readings, respectively. The results were expressed as $\mu \mathrm{mol}$ trolox equivalents per gram of sample on a dry weight basis.

\subsection{Statistical analysis}

A completely randomized design with three replications $(n=3)$ was used. The results were expressed as mean values \pm standard deviation. Data analysis was carried out using ANOVA, Tukey's test $(p<0.05)$ and Statistica 10.0 (StatSoft, Tulsa, OK, USA).

\section{Results and discussion}

\subsection{Effects of ultrasound and hemicellulase treatment on the activity of endogenous 6-glucosidase}

The hydrolysis of conjugated isoflavones with a paralle increase in their respective aglycones may be beneficial due to the higher bioacessibility of the latter ones in the small intestine. Therefore, consumption of soybean products showing hydrolised isoflavones may render improved health effects. Control soymilk (devoid of any treatment) showed B-glucosidase activity of $0.17 \pm$ $0.01 \mathrm{AU} \cdot \mathrm{g}^{-1}$ (Figure 1). As for the effects of different treatments, soymilk obtained upon HAE or UAE showed $1.3-(0.22 \pm 0.01$ $\left.\mathrm{AU} \cdot \mathrm{g}^{-1}\right)$ and 1.5 -fold $\left(0.26 \pm 0.02 \mathrm{AU} \cdot \mathrm{g}^{-1}\right)$ higher $\beta$-glucosidase activity than that of the control. Furthermore, all samples showed 
statistically different enzyme activities $(p<0.05)$. According to the literature (McClements, 1995), due to the intense pressures, temperatures and shear forces, high-intensity ultrasound and prolonged exposure may denature protein, thus causing inhibition of the enzyme catalytic activity. However, increased activity following short exposures to ultrasound has also been found. Furthermore, the same author also mentioned that ultrasound may be able to break down molecular aggregates, thus facilitating enzyme approach for the reaction to occur. Lending support to the statements made by McClements (1995), Ma et al. (2011) demonstrated that the activity of Alcalase was increased upon ultrasound treatment (up to $400 \mathrm{~W}$ ). However, such increasing trend was noted only until $80 \mathrm{~W}$. When ultrasonic intensity exceeded $80 \mathrm{~W}$, a decresing trend was then observed. The same authors also reported that the activity of Alcalase increased when the treatment $(80 \mathrm{~W})$ lasted for $4 \mathrm{~min}$, and longer exposure time decreased the enzyme activity. After $10 \mathrm{~min}$ of exposure the activity was lower than that of the control. Therefore, the effects of ultrasound treatment appear to be dependent on several parameters but this matter has not yet been entirely clarified. Formation of certain number of $\alpha$-helix structure, loss of $\beta$-sheet and decrease of random coil content are examples of conformational changes of the protein induced by ultrasound (Ma et al., 2011). Due to conformational changes, ultrasound treatment under optimized conditions (e.g. frequency, intensity, time of exposure) may be helpful to increase the activity of added enzymes and to improve the extraction and/or conversion of molecules of interest (Falcão et al., 2018; Ma et al., 2011; Nadar and Rathod, 2017).

\subsection{Isoflavone profile as affected by ultrasound and hemicellu- lase treatment}

Free radical generation from water molecules is among the mechanisms involved during ultrasound treatment (Delgado-Povedano and de Castro, 2015). Hence, oxidation of bioactive compounds may be contemplated. Furthermore, different phenolic antioxidants may respond differently to oxidative stress. Although the effect of soybean treatment with ultrasound prior to hydration has already been studied (Falcão et al., 2018), the high content of water present in the system during soymilk manufacturing may increase ultrasound-induced detrimental effects on phenolic antioxidants. Furthermore, several pieces of evidence have reported the decrease and/or degradation of antioxidant compounds such as vitamin C, flavonoids, and tocopherols upon ultrasound treatment (Clodoveo et al., 2013; Gamboa-Santos et al., 2013; Jahouach-Rabai et al., 2008; Moradi et al., 2018; Tiwari et al., 2010). Therefore, before industrial application, an in-depth chemical investigation is recommended.

Hemicellulase and ultrasound treatment decresed the content of malonyl or $\beta$-glucoside isoflavones. However, while the concentration decrease of $\beta$-glucoside isoflavones $(40 \%)$ was equal regardless of the treatment employed, but a higher decrease of malonyl isoflavones $(18 \%)$ was noted in soymilk obtained upon HAE compared to that of UAE $(9.9 \%)$. The same trend was observed for their respective aglycones. Furthermore, the lower decrease, and, potential conversion of malonyl isoflavones compared to those of $\beta$-glucoside isoflavones may be related to their structural characteristics. The malonyl group linked to the carbon six of the glucose moiety may induce ionic or steric hindrance of the hemicellulase, thus imparing its action (Ismael and Hayes, 2005).

In contrast, a positive effect was found in the aglycone group. The concentration of isoflavones in the aglycone form increased to 1.7 and 2.4-fold in the samples subjected to HAE and UAE, a Control

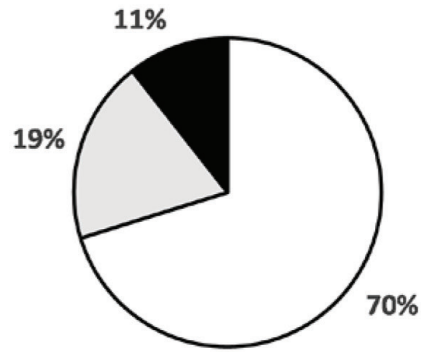

akalonylglucosides $\square \beta$-Glucosides aglycones

b

HAE

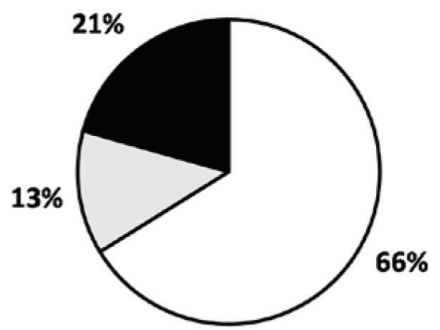

$\square$ Malonylglucosides $\square \beta$-Glucosides aglycones

C

UAE

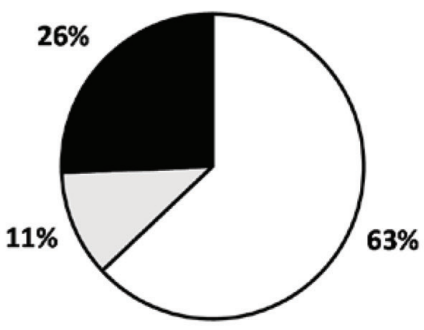

口Malonylglucosides $\square \beta$-Glucosides Aglycones

Figure 2. The contribuition of different forms of isoflavones in soymilk as affected by hemicellulase-assisted extraction (HAE) and ultrasoundassisted extraction (UAE).

respectively, compared to that of the control. Considering the total isoflavone content (Figure 2), the percentage of aglycones increased from 11 (control) to $26 \%$ (UAE). Therefore, it is clear that ultrasound treatment is the best option to recover isoflavones in the aglycone form. In addition, the efficiency of increasing the yield of aglycones upon ultrasound treatment was in the order of glycitein $>$ genistein $>$ daidzein.

The effect of thermal treatment of soybean flour on the conversion of isoflavones has already been reported (Andrade et al., 2016). However, conversion may not be the best term for the present study, especially in the case of HAE. Figure 3 displays the concentration changes found for each treatment. While the decrease in the concentration of conjugated isoflavones due to UAE rendered 
a $\square$ conjugated decrease $\quad \square$ aglycone increase
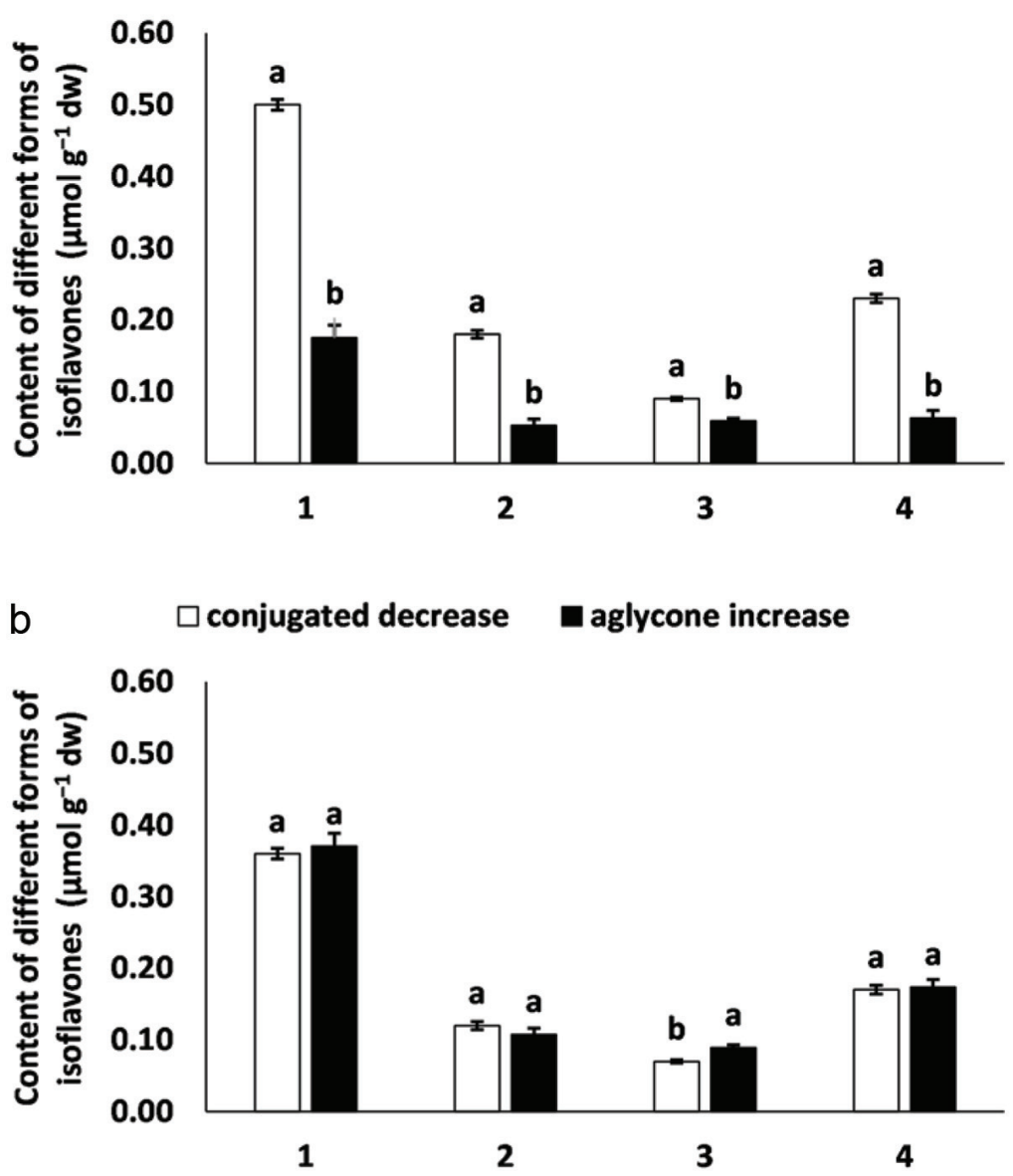

Figure 3. Concentration changes of different forms of isoflavones in soymilk as affected by hemicellulase-assisted extraction (a) and ultrasound-assisted extraction (b). \#1, 2, 3, and 4 (blank bars) are total conjugated isoflavones, conjugated daidzein, conjugated glycitein, and conjugated genistein, respectively. The same numbers (black bars) are total aglycone isoflavones, daidzein, glycitein, and genistein, respectively.

an equimolar increase in the total concentration of aglycones, the same was not observed for samples subjected to HAE. The exact reason for that is not clear and warrants further investigation.

In summary, the results of this contribution indicate that soymilk obtained under HAE and/or UAE show higher contents of isoflavones in the aglycone form. This may be explained by the hydrolysis of the $\beta-1,4$ linkage present between the glycosyl and malonyl moieties thus generating aglycones. However, due to the higher yield of isoflavone aglycones in the sample obtained under $\mathrm{UAE}$ as well as due to their equimolar conversion, ultrasound may provide the best option for the food industry.

\subsection{Antiradical activity as affected by ultrasound and hemicel- lulase treatment}

The mechanism of action of phenolic compounds has been explained by single electron transfer (SET) or hydrogen atom transfer (HAT) (de Camargo et al., 2017; Leopoldini et al., 2004; Yoshiara et al., 2018b). However, using a single method is insufficient to substantiate the potential antioxidant activity of the test material as well as to support the changes observed upon different food processing methods. Therefore, at least two different assays have been employed for such purposes. The ABTS method relies on SET and it is has been widely employed due to its application to hydrophilic and lipophilic compounds (de Camargo et al., 2017; Leopoldini et al., 2004; Yoshiara et al., 2018b). Furthermore, its short reaction time, usually $6 \mathrm{~min}$, and low amount of sample (e.g. 20-30 $\mu \mathrm{L}$ ), even when the reaction is not carried out in microplates, makes the ABTS one of the most important methods when limited amount of sample and/or compound is available (Oldoni et al., 2016). In addition, at least one method of biological relevance must be used. The oxygen radical absorbance capacity (ORAC) method evaluates the scavening activity of natural compounds, including (poly) phenols, towards peroxyl radicals. According to Granato et al. (2018), ORAC is based on HAT. It is well accepted that similar to other reactive oxygen species (ROS), peroxyl radicals are detrimental to lipid and protein, which makes them potentially harmful to biological systems. Peroxyl radicals may cause damage at cellular level as well as in components of biological fluids, and this has been at least partially explained by their relatively long half-life compared to that of hydroxyl radicals. Furthermore, a recent study by de Camargo et al. (2019a) demonstrated that the scavenging activity of phenolic compounds towards peroxyl radical was quite similar to the inhibition of NF-KB in RAW 264.7 macrophages pretreated with flavonoid-rich extracts. 


\section{$\square$ ABTS assay $\square$ ORAC assay}

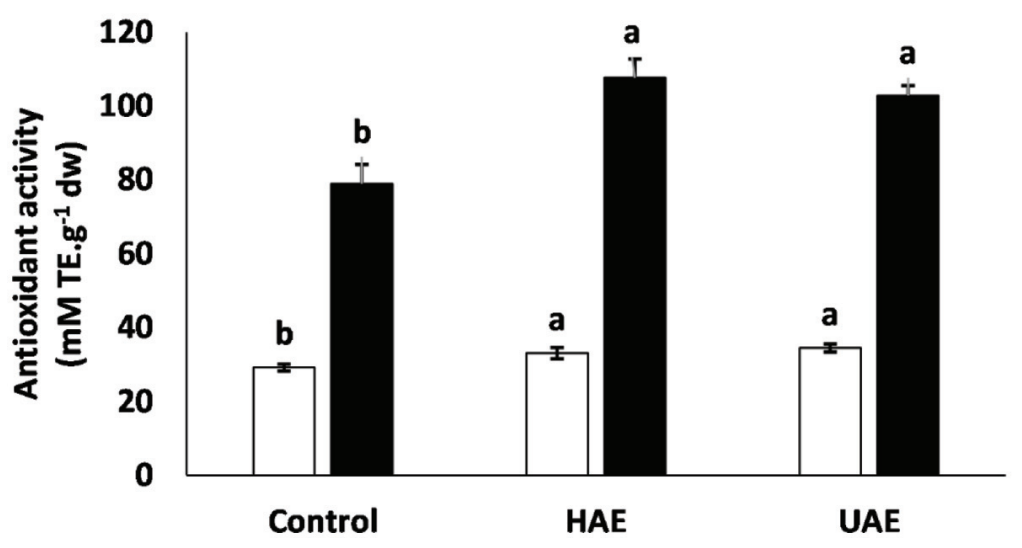

Figure 4. Antiradical activity of soymilk produced under hemicellulase-assisted extraction (HAE) and ultrasound-assisted extraction (UAE). Data represent the mean \pm standard deviation of each sample $(n=3)$. Means with different letters indicate significant differences $(p<0.05)$ among treatments, considering each assay.

In the present study, no difference was found between the samples obtained under HAE and UAE towards ABTS radical cation (Figure 4a) and peroxyl radical (Figure 4b). However, regardless of the method employed, both methods showed higher antiradical activity compared to that of the control. In general, such increase has been explained by the higher concentration of isoflavone aglycones present in the test sample (Table 1). However, the isoflavone profile of soybean and its processing products is well established. Therefore, considering this, we have proposed an index to anticipate the potential antioxidant activity of soybean products (Table 2). According to this index, a high positive correlation existed between the aglycone isoflavones/conjugated isoflavones ratio (aISO/cISO) and both radicals. Therefore, our results lend support to the importance of the structure-activity relationship.

Several studies have demonstrated that ROS can cause DNA strand breakage (Perera et al., 2018; Pohl et al., 2018; Rahman et al., 2017), which has been used as a biomarker in the screening of natural compounds that may reduce the risk of cancer development. Oxidative stess is common to many non-communicable diseases (NCDs) such as cardiovascular ailments, cancer, diabetes and obesity (Chiu et al., 2018; de Camargo et al., 2018). Furthermore, the ability of phenolic compounds in reducing inflammation is explained primarily by their antioxidant properties (Zhang and Tsao, 2016). Therefore, due to their improved antioxidant potential, soymilk procured under HAE and UAE may render improved health benefits by counteracting the aforementioned health issues. In addition, soymilk is used to produce many food products such as tofu, fermented soymilk, and soy yogurts, to name a few. Thus, soybean products produced with soymilk obtained under HAE and/or UAE may also render products with improved antioxidant

Table 1. Isoflavone profile ( $\mu \mathrm{mol} \mathrm{g}^{-1} \mathrm{dw}$ ) of soymilk obtained by conventional method (control), hemicellulase-assisted extraction (HAE), and ultrasoundassisted extraction (UAE)

\begin{tabular}{|c|c|c|c|}
\hline & control & HAE & UAE \\
\hline Malonylglucosides & $1.72 \pm 0.01^{a}$ & $1.41 \pm 0.02^{c}$ & $1.55 \pm 0.01^{b}$ \\
\hline malonyldaidzin & $0.57 \pm 0.00^{a}$ & $0.45 \pm 0.01^{c}$ & $0.51 \pm 0.01^{b}$ \\
\hline malonylglycitin & $0.25 \pm 0.00^{a}$ & $0.21 \pm 0.00^{c}$ & $0.23 \pm 0.00^{b}$ \\
\hline malonylgenistin & $0.91 \pm 0.00^{a}$ & $0.76 \pm 0.01^{c}$ & $0.82 \pm 0.00^{b}$ \\
\hline B-Glucosides & $0.47 \pm 0.01^{a}$ & $0.28 \pm 0.01^{b}$ & $0.28 \pm 0.00^{b}$ \\
\hline daidzin & $0.19 \pm 0.00^{a}$ & $0.12 \pm 0.01^{b}$ & $0.12 \pm 0.00^{b}$ \\
\hline glycitin & $0.12 \pm 0.00^{a}$ & $0.07 \pm 0.00^{b}$ & $0.07 \pm 0.00^{b}$ \\
\hline genistin & $0.16 \pm 0.00^{a}$ & $0.09 \pm 0.00^{b}$ & $0.09 \pm 0.00^{b}$ \\
\hline Aglycones & $0.26 \pm 0.01^{c}$ & $0.44 \pm 0.00^{b}$ & $0.63 \pm 0.04^{a}$ \\
\hline daidzein & $0.10 \pm 0.01^{c}$ & $0.15 \pm 0.00^{b}$ & $0.21 \pm 0.00^{a}$ \\
\hline glycitein & $0.05 \pm 0.00^{c}$ & $0.11 \pm 0.00^{b}$ & $0.14 \pm 0.00^{a}$ \\
\hline genistein & $0.11 \pm 0.02^{b}$ & $0.17 \pm 0.00^{b}$ & $0.28 \pm 0.04^{a}$ \\
\hline
\end{tabular}

Data represent mean values \pm standard deviation $(n=3)$. Values followed by the same letters within a column are not significantly different $(p>0.05)$. DW is dry weight. The contents ( $\mu \mathrm{mol} \mathrm{g}{ }^{-1} \mathrm{dw}$ ) of isoflavones in the original soybean were $1.84 \pm 0.27$ (malonylglucosides); $0.62 \pm 0.03$ (malonyldaidzin); $0.34 \pm 0.01$ (malonylglycitin); $0.88 \pm 0.02$ (malonylgenistin); $0.71 \pm 0.02$ ( $\beta$-glucosides); $0.26 \pm 0.01$ (daidzin); $0.22 \pm 0.01$ (glycitin); $0.23 \pm 0.01$ (genistin); $0.17 \pm 0.00$ (aglycones); $0.06 \pm 0.00$ (daidzein); $0.06 \pm 0.00$ (glyctein); $0.06 \pm 0.00$ (genistein). 
Table 2. Aglycone to conjugated isoflavone ratio as an index of antioxidant potential

\begin{tabular}{lllll}
\hline & \multicolumn{3}{c}{ Ratio } & aGEN/cGEN \\
\cline { 2 - 4 } & alSO/clSO & aDAl/cDAl & aGLY/cGLY & $0.053 \pm 0.001^{\mathrm{d}}$ \\
\hline feedstock & $0.068 \pm 0.003^{\mathrm{d}}$ & $0.063 \pm 0.002^{\mathrm{d}}$ & $0.105 \pm 0.005^{\mathrm{d}}$ & $0.102 \pm 0.015^{\mathrm{c}}$ \\
control & $0.120 \pm 0.006^{\mathrm{c}}$ & $0.132 \pm 0.009^{\mathrm{c}}$ & $0.146 \pm 0.005^{\mathrm{c}}$ & $0.204 \pm 0.000^{\mathrm{b}}$ \\
HAE & $0.258 \pm 0.002^{\mathrm{b}}$ & $0.268 \pm 0.003^{\mathrm{b}}$ & $0.404 \pm 0.005^{\mathrm{b}}$ & $0.314 \pm 0.046^{\mathrm{a}}$ \\
UAE & $0.345 \pm 0.021^{\mathrm{a}}$ & $0.327 \pm 0.005^{\mathrm{a}}$ & $0.475 \pm 0.005^{\mathrm{a}}$ & \\
\hline
\end{tabular}

\begin{tabular}{|c|c|c|c|c|}
\hline \multirow[b]{2}{*}{ ORAC } & \multicolumn{4}{|c|}{ Correlation } \\
\hline & $0.8846^{*}$ & $0.9273^{*}$ & 0.8899* & $0.8332 *$ \\
\hline ABTS & $0.8471^{*}$ & $0.8860 *$ & $0.8436 *$ & $0.8053^{*}$ \\
\hline
\end{tabular}

Abbreviations: alSO, aglycone isoflavones; cISO, conjugated isoflavones; aDAl, daidzein; cDAl, conjugated daidzein; aGly, glycitein; cGly, conjugated glycitein; aGEN, genistein cGEN, conjugated genistein; Data represent mean values \pm standard deviation $(n=3)$. Values followed by the same letters within a column are not significantly different $(p>0.05)$. *significant at $p<0.05$.

properties.

\section{Conclusion}

Both hemicellulase assisted- and ultrasound-assisted-extraction were demonstrated to influence the activity of endogenous $\beta$-glucosidase, the isoflavone profile and the antioxidant potential of soymilk. Endogenous $\beta$-glicosidase activity and the contents of isoflavone aglycones were in the decreasing order of ultrasoundassisted extraction $>$ hemicellulase-assisted extraction $>$ control. The present contribution supports the use of ultrasound-assisted extraction to achieve an equimolar conversion from conjugated isoflavones into their respective aglycones. However, further investigations are needed to clarify the reasons behind the efficacy of ultrasound as a novel technology. No differences were found between the antiradical activity of ultrasound-assisted extraction and hemicellulase-assisted extraction, both of which showed higher effects than that of the control. The ratio of aglycone isoflavones to conjugated isoflavones may be used as an index of antioxidant potential and/or quality for such commodities.

\section{Acknowledgments}

M.B.R. Silva (Ph.D fellowship), H.G. Falcão (Ph.D. fellowship), and A.C. de Camargo (Postdoctoral fellowship) acknowledge CAPES (Coordenação de Aperfeiçoamento de Pessoal de Nível Superior - Brazil). One of us (F. Shahidi) thanks the Natural Science and Engineering Research Council (NSERC) of Canada for partial financial support. E.I. Ida is a CNPq research fellow (process \#305397/2015-0).

\section{Author contributions}

M.B.R. Silva and H.G. Falcão were responsible for the soymilk preparation, chemical analysis, and wrote the first version of the manuscript. L.E. Kurozawa, S.H. Prudencio, and E.I. Ida provided experimental design, and supervised the soymilk preparation, and chemical analysis. M.B.R. Silva, A.C. de Camargo, and E.I. Ida were responsible for statistical analysis and interpreted the data. A.C. de Camargo and E.I. Ida wrote the final manuscript. A.C. de
Camargo, F. Shahidi, and E.I. Ida proposed the new quality index of antioxidant potential and/or quality for soybean and its processed products. E.I. Ida and F. Shahidi supervised data interpretation and edited the final manuscript.

\section{References}

Andrade, J.C., Mandarino, J.M.G., Kurozawa, L.E., and Ida, E.I. (2016). The effect of thermal treatment of whole soybean flour on the conversion of isoflavones and inactivation of trypsin inhibitors. Food Chem. 194: 1095-1101.

Arvayo-Enríquez, H., Mondaca-Fernández, I., Gortárez-Moroyoqui, P., López-Cervantes, J., and Rodríguez-Ramírez, R. (2013). Carotenoids extraction and quantification: a review. Anal. Methods 5: 2916-2924.

Baú, T.R., Garcia, S., and Ida, E.I. (2015). Changes in soymilk during fermentation with kefir culture: oligosaccharides hydrolysis and isoflavone aglycone production. Int. J. Food Sci. Nutr. 66: 845-850.

Baú, T.R., and Ida, E.I. (2015). Soymilk processing with higher isoflavone aglycone content. Food Chem. 183: 161-168.

Chiu, H.-F., Shen, Y.-C., Venkatakrishnan, K., and Wang, C.-K. (2018). Popular functional foods and nutraceuticals with lipid lowering activity and in relation to cardiovascular disease, dyslipidemia, and related complications: an overview. J. Food Bioact. 2: 16-27.

Clodoveo, M.L., Durante, V., and La Notte, D. (2013). Working towards the development of innovative ultrasound equipment for the extraction of virgin olive oil. Ultrason Sonochem. 20: 1261-1270.

de Camargo, A.C., Biasoto, A.C.T., Schwember, A.R., Granato, D., Rasera, G.B., Franchin, M., Rosalen, P.L., Alencar, S.M., and Shahidi, F. (2019a). Should we ban total phenolics and antioxidant screening methods? The link between antioxidant potential and activation of NF-kB using phenolic compounds from grape by-products. Food Chem. 290: 229-238.

de Camargo, A.C., Favero, B.T., Morzelle, M.C., Franchin, M., Alvarez-Parrilla, E., de la Rosa, L.A., Geraldi, M.V., Maróstica Júnior, M.R., Shahidi, F., and Schwember, A.R. (2019b). Is chickpea a potential substitute for soybean? Phenolic bioactives and potential health benefits. Int. J. Mol. Sci. 20: 2644.

de Camargo, A.C., Regitano-d'Arce, M.A.B., and Shahidi, F. (2017). Phenolic profile of peanut by-products: antioxidant potential and inhibition of alpha-glucosidase and lipase activities. J. Am. Oil Chem. Soc. 94: 959-971.

de Camargo, A.C., Regitano-d'Arce, M.A.B., Biasoto, A.C.T., and Shahidi, F. (2016). Enzyme-assisted extraction of phenolics from winemaking by-products: antioxidant potential and inhibition of alpha-glucosidase and lipase activities. Food Chem. 212: 395-402.

de Camargo, A.C., Schwember, A.R., Parada, R., Garcia, S., Maróstica 
Junior, M.R., Franchin, M., Regitano-d'Arce, M.A.B., and Shahidi, F. (2018). Opinion on the hurdles and potential health benefits in value-added use of plant food processing by-products as sources of phenolic compounds. Int. J. Mol. Sci. 19: 3498.

de Lima, F.S., and Ida, E.I. (2014). Optimisation of soybean hydrothermal treatment for the conversion of $\beta$-glucoside isoflavones to aglycones. LWT - Food Sci. Technol. 56: 232-239.

Delgado-Povedano, M.M., and de Castro, M.D.L. (2015). A review on enzyme and ultrasound: A controversial but fruitful relationship. Anal. Chim. Acta. 889: 1-21.

Falcão, H.G., Handa, C.L., Silva, M.B.R., de Camargo, A.C., Shahidi, F., Kurozawa, L.E., and Ida, E.I. (2018). Soybean ultrasound pre-treatment prior to soaking affects $\beta$-glucosidase activity, isoflavone profile and soaking time. Food Chem. 269: 404-412.

Farzamirad, V., and Aluko, R.E. (2008). Angiotensin-converting enzyme inhibition and free-radical scavenging properties of cationic peptides derived from soybean protein hydrolysates. Int. J. Food Sci. Nutr. 59: 428-437.

Fernandes, M.S., de Lima, F.S., Rodrigues, D., Handa, C., Guelfi, M., Garcia, S., and Ida, E.I. (2017). Evaluation of the isoflavone and total phenolic contents of kefir-fermented soymilk storage and after the in vitro digestive system simulation. Food Chem. 229: 373-380.

Gamboa-Santos, J., Cristina Soria, A., Pérez-Mateos, M., Carrasco, J.A., Montilla, A., and Villamiel, M. (2013). Vitamin C content and sensorial properties of dehydrated carrots blanched conventionally or by ultrasound. Food Chem. 136: 782-788.

Granato, D., Shahidi, F., Wrolstad, R., Kilmartin, P., Melton, L.D., Hidalgo, F.J., Miyashita, K., Camp, J.V., Alasalvar, C., Ismail, A.B., Elmore, S., Birch, G.G., Charalampopoulos, D., Astley, S.B., Pegg, R., Zhou, P., and Finglas, P. (2018). Antioxidant activity, total phenolics and flavonoids contents: Should we ban in vitro screening methods? Food Chem. 264: 471-475.

Handa, C.L., Couto, U.R., Vicensoti, A.H., Georgetti, S.R., and Ida, E.I. (2014). Optimisation of soy flour fermentation parameters to produce $\beta$-glucosidase for bioconversion into aglycones. Food Chem. 152: 56-65.

Handa, C.L., de Lima, F.S., Guelfi, M.F.G., Georgetti, S.R., and Ida, E.I. (2016). Multi-response optimisation of the extraction solvent system for phenolics and antioxidant activities from fermented soy flour using a simplex-centroid design. Food Chem. 197: 175-184.

Ismael, B., and Hayes, K. (2005). $\beta$-Glycosidase activity toward different glycosidic forms of isoflavones. J. Agric. Food Chem. 53: 4918-4924.

Izumi, T., Piskula, M.K., Osawa, S., Obata, A., Tobe, K., Saito, M., and Kikuchi, M. (2000). Soy isoflavone aglycones are absorbed faster and in higher amounts than their glucosides in humans. J. Nutr. 130: 1695-1699.

Jahouach-Rabai, W., Trabelsi, M., Van Hoed, V., Adams, A., Verhé, R., De Kimpe, N., and Frikha, M.H. (2008). Influence of bleaching by ultrasound on fatty acids and minor compounds of olive oil. Qualitative and quantitative analysis of volatile compounds (by SPME coupled to GC/MS). Ultrason Sonochem. 15: 590-597.

Juturu, V., and Wu, J.C. (2012). Insight into microbial hemicellulases other than xylanases: a review. J. Chem. Technol. Biotechnol. 88: 353-363.

Kalaiselvan, V., Kalaivani, M., Vijayakumar, A., Sureshkumar, K., and Venkateskumar, K. (2010). Current knowledge and future direction of research on soy isoflavones as a therapeutic agents. Pharmacogn Rev. 4: 111-117.

Leopoldini, M., Marino, T., Russo, N., and Toscano, M. (2004). Antioxidant properties of phenolic compounds: $\mathrm{H}$-atom versus electron transfer mechanism. J. Phys. Chem. A 108: 4916-4922.

Ma, H., Huang, L., Jia, J., He, R., Luo, L., and Zhu, W. (2011). Effect of energygathered ultrasound on Alcalase. Ultrason Sonochem. 18: 419-424.

Matsuura, M., and Obata, A. (1993). $\beta$-Glucosidases from soybeans hydrolyze daidzin and genistin. J. Food Sci. 58: 144-147.
McClements, D.J. (1995). Advances in the application of ultrasound in food analysis and processing. Trends Food Sci. Technol. 6: 293-299.

Melo, P.S., Massarioli, A.P., Denny, C., dos Santos, L.F., Franchin, M., Pereira, G.E., Vieira, T.M.F.S., Rosalen, P.L., and Alencar, S.M. (2015). Winery by-products: Extraction optimization, phenolic composition and cytotoxic evaluation to act as a new source of scavenging of reactive oxygen species. Food Chem. 181: 160-169.

Moradi, N., Rahimi, M., Moeini, A., and Parsamoghadam, M.A. (2018). Impact of ultrasound on oil yield and content of functional food ingredients at the oil extraction from sunflower. Sep. Sci. Technol. 53: 261-276.

Nadar, S.S., and Rathod, V.K. (2017). Sonochemical effect on activity and conformation of commercial lipases. Appl. Biochem. Biotechnol. 181: 1435-1453.

Oldoni, T.L.C., Melo, P.S., Massarioli, A.P., Moreno, I.A.M., Bezerra, R.M.N., Rosalen, P.L., da Silva, G.V.J., Nascimento, A.M., and Alencar, S.M (2016). Bioassay-guided isolation of proanthocyanidins with antioxidant activity from peanut (Arachis hypogaea) skin by combination of chromatography techniques. Food Chem. 192: 306-312.

Perera, N., Ambigaipalan, P., and Shahidi, F. (2018). Epigallocatechin gallate (EGCG) esters with different chain lengths fatty acids and their antioxidant activity in food and biologicalsystems. J. Food Bioact. 1: 124-133.

Pohl, F., Goua, M., Bermano, G., Russell, W.R., Scobbie, L., Maciel, P., and Kong Thoo Lin, P. (2018). Revalorisation of rapeseed pomace extracts: An in vitro study into its anti-oxidant and DNA protective properties. Food Chem. 239: 323-332.

Preece, K.E., Hooshyar, N., Krijgsman, A., Fryer, P.J., and Zuidam, N.J. (2017). Intensified soy protein extraction by ultrasound. Chem. Eng. Process. 113: 94-101.

Rahman, M.J., de Camargo, A.C., and Shahidi, F. (2017). Phenolic and polyphenolic profiles of chia seeds and their in vitro biological activities. J. Funct. Foods 35: 622-634.

Rao, P.R., and Rathod, V.K. (2015). Mapping study of an ultrasonic bath for the extraction of andrographolide from Andrographis paniculata using ultrasound. Ind. Crops Prod. 66: 312-318.

Re, R., Pellegrini, N., Proteggente, A., Pannala, A., Yang, M., and Rice-Evans, C. (1999). Antioxidant activity applying an improved ABTS radical cation decolorization assay. Free Radic. Biol. Med. 26: 1231-1237.

Riaz, M.N. (2006). Processing of soybeans into ingredients. Soy applications in food. In: Riaz, M.N. (Ed.). CRC: Taylor \& Francis, Boca Raton, FL, pp. 39-62.

Shahidi, F., and Peng, H. (2018). Bioaccessibility and bioavailability of phenolic compounds. J. Food Bioact. 4: 11-68.

Tiwari, B.K., Patras, A., Brunton, N., Cullen, P.J., and O'Donnell, C.P. (2010). Effect of ultrasound processing on anthocyanins and color of red grape juice. Ultrason Sonochem. 17: 598-604.

Toro-Funes, N., Bosch-Fusté, J., Veciana-Nogués, M.T., and Vidal-Carou, M.C. (2014). Effect of ultra high pressure homogenization treatment on the bioactive compounds of soya milk. Food Chem. 152: 597-602.

Veitch, N.C. (2007). Isoflavonoids of the Leguminosae. Nat. Prod. Rep. 24: 417-464.

Yoshiara, L., Madeira, T., de Camargo, A., Shahidi, F., and Ida, E. (2018a). Multistep optimization of $\beta$-glucosidase extraction from germinated soybeans (Glycine max L. Merril) and recovery of isoflavone aglycones. Foods 7: 110.

Yoshiara, L.Y., Mandarino, J.M.G., Carrão-Panizzi, M.C., Madeira, T.B., da Silva, J.B., de Camargo, A.C., Shahidi, F., and Ida, E.I. (2018b). Germination changes the isoflavone profile and increases the antioxidant potential of soybean. J. Food Bioact. 3: 144-150.

Zhang, H., and Tsao, R. (2016). Dietary polyphenols, oxidative stress and antioxidant and anti-inflammatory effects. Curr. Opin. Food Sci. 8: 33-42. 